\title{
Analytical and Numerical Solutions of Pollution Concentration with Uniformly and Exponentially Increasing Forms of Sources
}

\author{
N. Manitcharoen (iD) and B. Pimpunchat \\ Department of Mathematics, Faculty of Science, King Mongkut's Institute of Technology Ladkrabang, Bangkok 10520, Thailand \\ Correspondence should be addressed to B. Pimpunchat; busayamas.pi@kmitl.ac.th
}

Received 8 August 2019; Revised 24 December 2019; Accepted 13 March 2020; Published 1 April 2020

Academic Editor: Mariano Torrisi

Copyright (c) 2020 N. Manitcharoen and B. Pimpunchat. This is an open access article distributed under the Creative Commons Attribution License, which permits unrestricted use, distribution, and reproduction in any medium, provided the original work is properly cited.

\begin{abstract}
The study of pollution movement is an important basis for solving water quality problems, which is of vital importance in almost every country. This research proposes the motion of flowing pollution by using a mathematical model in one-dimensional advection-dispersion equation which includes terms of decay and enlargement process. We are assuming an added pollutant sources along the river in two cases: uniformly and exponentially increasing terms. The unsteady state analytical solutions are obtained by using the Laplace transformation, and the finite difference technique is utilized for numerical solutions. Solutions are compared by relative error values. The result appears acceptable between the analytical and numerical solutions. Varying the value of the rate of pollutant addition along the river $(q)$ and the arbitrary constant of exponential pollution source term $(\lambda)$ is displayed to explain the behavior of the incremental concentration. It is shown that the concentration increases as $q$ and $\lambda$ increase, and the exponentially increasing pollution source is a suitable model for the behavior of incremental pollution along the river. The results are presented and discussed graphically. This work can be applied to other physical situations described by advection-dispersion phenomena which are affected by the increase of those source concentrations.
\end{abstract}

\section{Introduction}

One of the major problems for area-connected water resources is water pollution. The source of this problem stems from the expansion of the community, the increase of industrial plants, and the increase of agricultural farms. Rivers carry water and nutrients to areas all around them, which is why contaminated water is having an impact on human health and is the leading cause of disease worldwide. Sources of water pollution occur due to the disposal of untreated point and nonpoint source pollutants. Point-source pollution refers to the pollutants discharged into a river from one discrete location or point, such as industrial or residential wastewater. The indicator used to measure the amount of pollution in water is biochemical oxygen demand (BOD). In this study, it is assumed that pollutants are largely biochemical waste and use the parameters from the Tha Chin river, a particular river in Thailand, as per Pimpunchat et al. (2007) [1, 2].
In Thailand, the Tha Chin river is one of the country's most polluted river. It has a continuously enlarged BOD. Agriculture is, apparently, the most important source of BOD loads affecting BOD concentrations along the river, followed by aquaculture, swine farms, rice cultivation, and agro-industry. Previous papers on the water quality of the Tha Chin river found that the lower part of the river was degraded, and that several major parameters exceeded the National Surface Water Quality Standards and Classification limits. The major water quality problems were low dissolved oxygen (DO), high ammonia nitrogen, high fecal coliform bacteria, high turbidity, and high organic matter (BOD). The degradation of water quality in rivers has affected the water quality and natural resources of the Gulf of Thailand. [3-5]. Figure 1 shows the monitoring of BOD for the third quarter of the year (April-June) between 2011 and 2018; it plotted from raw data that obtained from the mobile application developed by Regional Environmental Office 5 [6]. It 


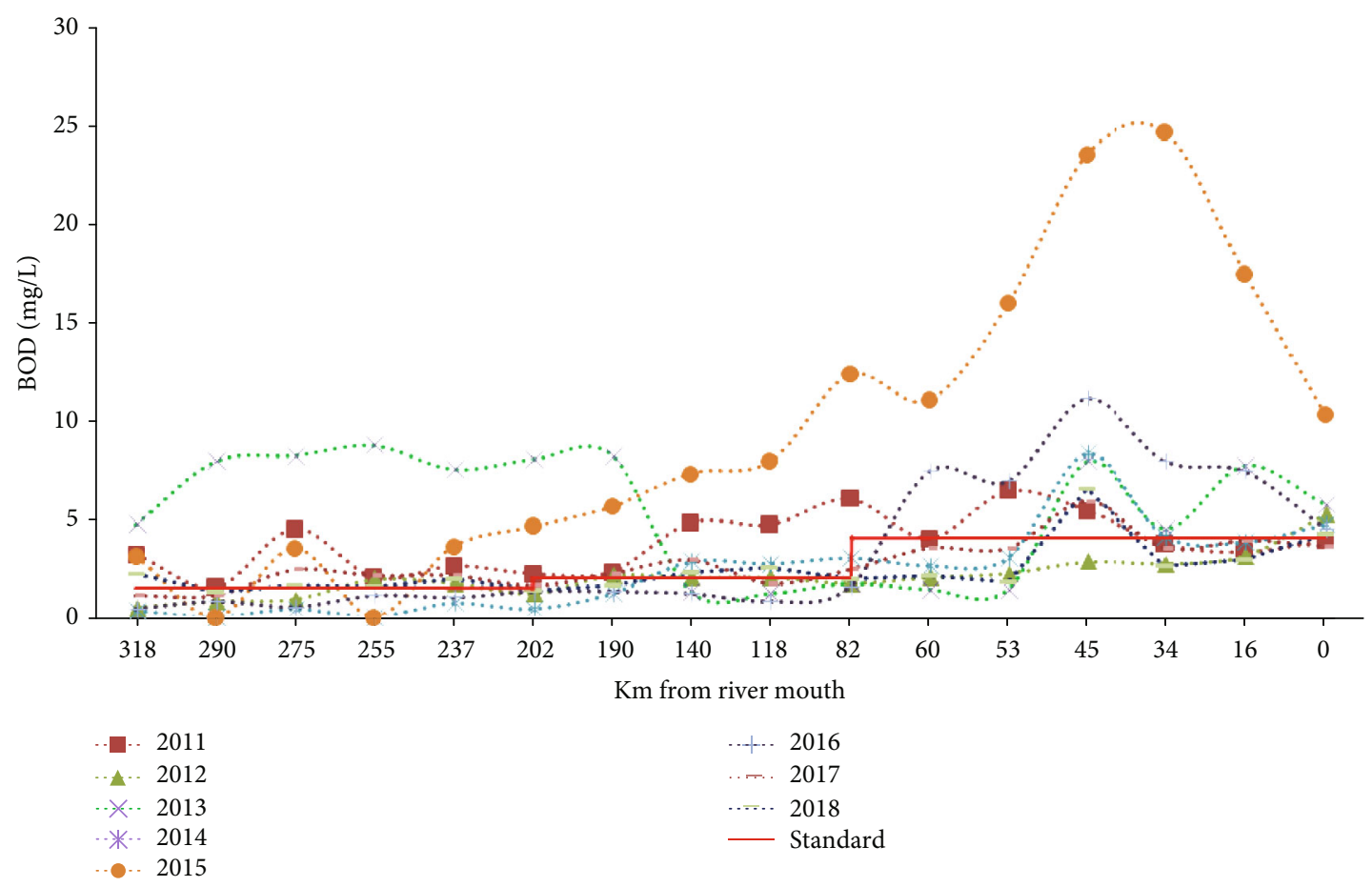

FIgUre 1: BOD status in the Tha Chin river (April-June) during 2011-2018. (Modified from raw data which is available from [6]).

indicates that BOD concentrations repeatedly exceeded the Class 4 BOD standards $(2-4 \mathrm{mg} / \mathrm{L})$ throughout the river $[7,8]$. Understanding the movement of pollution is useful for controlling and predicting water quality in rivers. For the assessment of the transport of contaminant changes in water quality (e.g., BOD), an advection-dispersion equation (ADE) is commonly used. ADE is a partial differential equation (PDE) derived from the mass balance applied to a mass volume unit in a river. The solution includes both analytical solutions and numerical solutions, based on the complications of the model. The Laplace transform technique has been widely applied to develop analytical solutions to ADE. Previous works in which ADE has been used in analysis include M.Th. Van Genuchten and W.J. Alves (1982), who presented analytical solutions of a one-dimensional convective-dispersive solute transport equation under a variety of conditions [9]; Kumar et al. (2010), who obtained analytical solutions for temporally and spatially dependent solute dispersion in a one-dimensional semi-infinite porous medium by using Laplace transform technique [10]; S. Savovic' and A. Djordjevich (2012), who described numerical solution by using the finite difference method for the 1-D advection diffusion partial differential equation with variable coefficients in semi-infinite media [11]; and Pimpunchat et al., who studied a mathematical model of pollution in river, presenting a simple model and providing some analytical solutions at steady state flows $[1,2]$.

In this study, the objective is to propose unsteady state solutions of the pollutant concentration by considering advection-dispersion equations in one dimension which includes terms of decay and increasing sources. Increasing sources were analysed by assuming the rate of pollutant addition along the river: $q$ in two cases and uniformly increasing and exponentially increasing forms. We approximate the arbitrary constants: $\lambda$ of exponentially increasing pollution source terms for predicting the behavior of concentration movement. Analytical solutions are obtained by using the Laplace transform technique and comparing the results by relative error with the numerical solution by using the explicit finite difference technique.

\section{Materials and Methods}

2.1. The Governing Equation. The unsteady flow in the river is modeled as 1-D using a single spatial $x(m)$ to describe the distance down the river from its source. Quantities, such as pollutants or oxygen concentrations, are only allowed to vary along the length of the river and are treated as homogeneous across the river cross-section. This assumption is justified by fulfilling Dobbin's criterion [12]. We use a single quantity to measure water pollution; the concentration of the pollutant $P(x, t)\left(\mathrm{kg} \mathrm{m}^{-3}\right)$ is assumed to vary with time $t$ (day). This is in the same form as a water pollution equation, 2.1, presented by Pimpunchat et al. [2]. The rate of change of the concentration with position $x$ and time $t$ are expressed as

$$
\begin{aligned}
\frac{\partial(A P)}{\partial t}= & D_{p} \frac{\partial^{2}(A P)}{\partial x^{2}}-\frac{\partial(v A P)}{\partial x}-K_{1} \frac{X}{X+k} A P \\
& +q H(x),(0 \leq x<L \leq \infty, t>0),
\end{aligned}
$$

where $H(x)$ is the Heaviside function

$$
H(x)= \begin{cases}1, & 0<x<L, \\ 0, & \text { otherwise }\end{cases}
$$




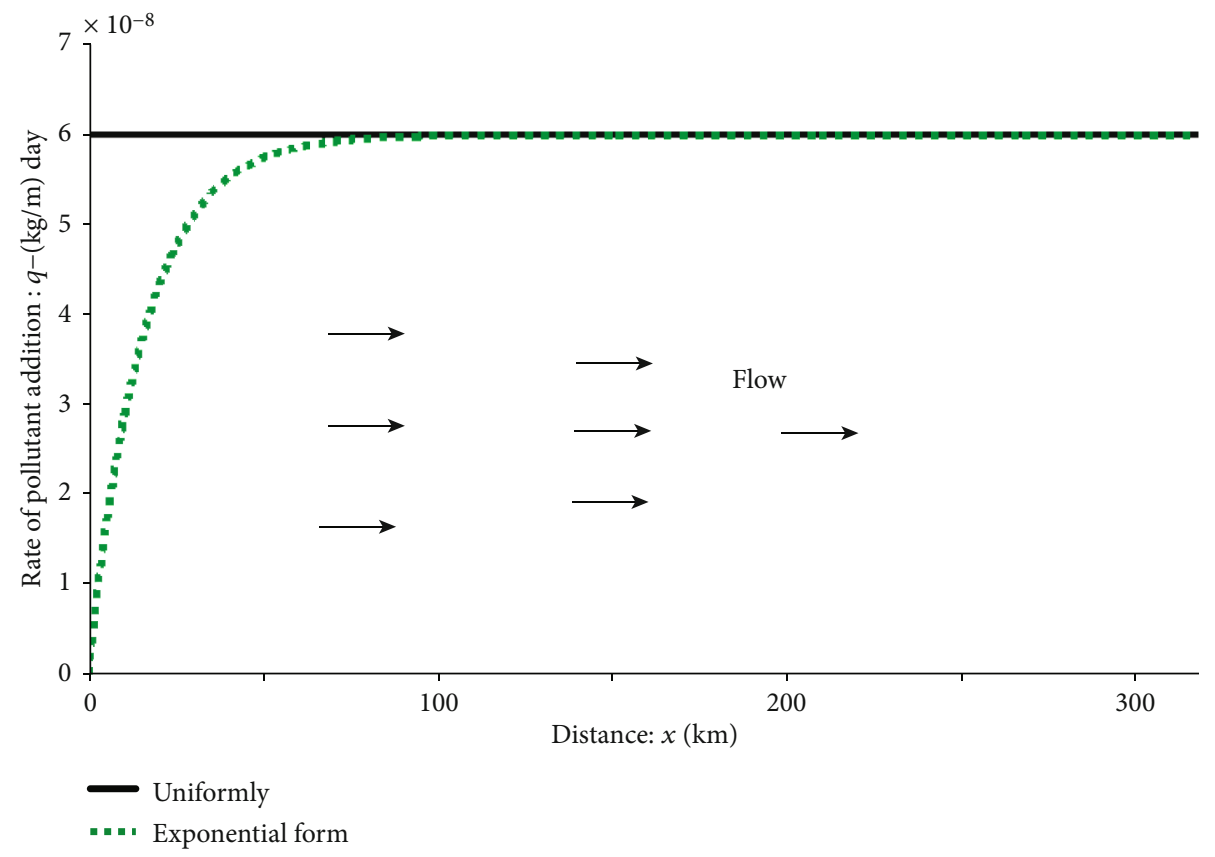

FIgURe 2: Physical system of the rate of pollutant addition $q$.

this equation is standard and was develop in Chapra [13]. We consider a river where pollutants are discharged in the form of wastes. It is assumed that these pollutants use dissolved oxygen $X(x, t)$ for various biochemical and biodegradation processes. The discharge of pollutants into the river is at the constant rate $q, A$ is the cross-section area of the river, $D_{p}$ is the dispersion coefficient of pollutant in the $x$ direction, $v$ is the water velocity in the $x$ direction, and $K_{1}$ is the degradation rate coefficient for pollutants. For convenience, all parameters, such as $A, D_{p}, v, K_{1}$, and $q$, hold constant values over time and space. Analysis is considered by the case of negligible $k(k \approx 0)$, and pollution sources are considered in two cases, uniformly increasing $P_{1}(x, t)$ and exponentially increasing pollution sources $P_{2}(x, t)$, demonstrated by Equations (3) and (4), respectively

$$
\begin{aligned}
\frac{\partial\left(A P_{1}\right)}{\partial t}= & D_{p} \frac{\partial^{2}\left(A P_{1}\right)}{\partial x^{2}}-\frac{\partial\left(v A P_{1}\right)}{\partial x}-K_{1} A P_{1}+q H(x) \\
\frac{\partial\left(A P_{2}\right)}{\partial t}= & D_{p} \frac{\partial^{2}\left(A P_{2}\right)}{\partial x^{2}}-\frac{\partial\left(v A P_{2}\right)}{\partial x}-K_{1} A P_{2} \\
& +q(1-\exp (-\lambda x)) H(x)
\end{aligned}
$$

where $\lambda$ is an arbitrary constant of exponential pollution source terms. The exponentially increasing form of pollution sources Equation (4) assumed by, the referred papers was found that the downstream pollution of the Tha Chin river is higher than the upstream, which is caused by geography, including various contributions from branch river to river and the increasing wastewater that comes from swine and rice farming [4-6]. Figure 2 sketches the physical system of the pollution sources described in Equations (3) and (7).
Let the domain be supposedly initially solute free, thus, initial conditions are

$$
P(x, t)=0, x \geq 0, t=0 .
$$

Boundary conditions at the origin of domain are considered by the uniformly increasing source concentration. The concentration gradient at the infinity is assumed to be zero. Then, boundary conditions associated with Equation (3) in a semi-infinite domain are as follows;

$$
\begin{gathered}
P(x, t)=P_{0}, x=0, t>0, \\
\frac{\partial P}{\partial x}(x, t)=0, x \longrightarrow \infty, t>0,
\end{gathered}
$$

where $P_{0}$ is source concentration at the origin.

2.2. Analytical Technique. Given a function $P(x, t)$ defined for all $t>0$ and assumed to be bounded, the Laplace transform in $t$ considering $x$ as parameter can be applied to Equation $(8)[14,15]$;

$$
\bar{P}(x, s)=L\{P(x, s) ; t \longrightarrow s\}=\int_{0}^{\infty} e^{-s t} P(x, t) d t,
$$

where $s$ is called the transform variable. Applying Laplace transformation to Equations (3) and (4) then, we get

$$
\begin{aligned}
D_{p} A & \frac{d^{2} \overline{P_{1}}(x, s)}{d x^{2}}-v A \frac{d \overline{P_{1}}(x, s)}{d x}-K_{1} A \bar{P}(x, s) \\
- & A\left(s \overline{P_{1}}(x, s)-P_{1}(x, 0)\right)+\frac{q}{s} \\
& =0, x \geq 0, s>0,
\end{aligned}
$$




$$
\begin{aligned}
D_{p} A & \frac{d^{2} \overline{P_{2}}(x, s)}{d x^{2}}-v A \frac{d \overline{P_{2}}(x, s)}{d x}-K_{1} A \overline{P_{2}}(x, s) \\
- & A\left(s \overline{P_{2}}(x, s)-P_{2}(x, 0)\right)+\frac{q}{s}(1-\exp (-\lambda x)) \\
& =0, x \geq 0, s>0 .
\end{aligned}
$$

Transformed initial and boundary conditions on Equations (5), (6), and (7) give

$$
\begin{aligned}
& \bar{P}(x, 0)=0, \\
& \bar{P}(0, s)=\frac{P_{0}}{s},
\end{aligned}
$$

and

$$
\frac{d \bar{P}}{d x}(x, s)=0 \text { as } x \longrightarrow \infty .
$$

Evaluating the solutions of Equations (9) and (10) by using initial and boundary conditions. Equations (11), (12), and (13) provide their solutions in the Laplace domain, which may be written as

$$
\begin{aligned}
\bar{P}_{1}(x, s)= & \left(\frac{P_{0}}{s}-\frac{q}{A s\left(s+K_{1}\right)}\right) \exp \left(\left(\gamma-\sqrt{\frac{s+\beta^{2}}{D_{p}}}\right) x\right) \\
& +\frac{q}{A s\left(s+K_{1}\right)}, \\
\bar{P}_{2}(x, s)= & \left(\frac{P_{0}}{s}-\frac{q}{A s\left(s+K_{1}\right)}+\frac{q}{A s\left(s+K_{3}\right)}\right) \exp \\
& \cdot\left(\left(\gamma-\sqrt{\frac{s+\beta^{2}}{D_{p}}}\right) x\right)+\frac{q}{A s\left(s+K_{1}\right)} \\
& -\frac{q \exp (-\lambda x)}{A s\left(s+K_{3}\right)},
\end{aligned}
$$

where $\gamma=v / 2 D_{p}, \beta=\sqrt{v^{2} / 4 D_{p}+K_{1}}$, and $K_{3}=K_{1}-v \lambda-$ $D_{p} \lambda^{2}$.

2.3. Numerical Technique. In this section, we present the numerical technique, in order to use the explicit finite difference technique by using the forward differences scheme for the time and the central derivatives for the space. Thus, Equations (3) and (7) in the finite difference form can be written as

$$
\begin{aligned}
\frac{P_{1 m}^{n+1}-P_{1 m}^{n}}{\Delta t}= & \frac{D_{p}}{\Delta x^{2}}\left(P_{1 m+1}^{n}-2 P_{1 m}^{n}+P_{1 m-1}^{n}\right) \\
& -\frac{v}{2 \Delta x}\left(P_{1 m+1}^{n}-P_{1 m-1}^{n}\right)-K_{1} P_{1 m}^{n} \\
& +\frac{q}{A}+0\left(\Delta x^{2}, \Delta t\right),
\end{aligned}
$$

$$
\begin{aligned}
\frac{P_{2 m}^{n+1}-P_{2 m}^{n}}{\Delta t}= & \frac{D_{p}}{\Delta x^{2}}\left(P_{2 m+1}^{n}-2 P_{2 m}^{n}+P_{2 m-1}^{n}\right) \\
& -\frac{v}{2 \Delta x}\left(P_{2 m+1}^{n}-P_{2 m-1}^{n}\right)-K_{1} P_{2 m}^{n}\left(\Delta x^{2}, \Delta t\right) \\
& +\frac{q}{A}\left(1-\exp \left(-\lambda x_{m}^{n}\right)\right)+0
\end{aligned}
$$

where indexes $m$ and $n$ refer to the discrete step size $\Delta x$ and the time step size $\Delta t$, respectively. The initial condition (4) and boundary conditions (5) and (6) for Equations (3) and (7) can be expressed in the finite difference form as;

$$
\begin{aligned}
P_{m, 0} & =0, x \geq 0, \\
P_{0, n} & =P_{0}, t>0, \\
P_{M, n} & =P_{M-1, n}, x \longrightarrow \infty, t \geq 0 .
\end{aligned}
$$

\section{Results and Discussions}

3.1. Analytical Solutions. The solutions of the Equations (14) and (15) are found by inverting the Laplace transform denoted by the complex variable Equation (16).

$$
\begin{aligned}
P(x, t) & =L^{-1}\{\bar{P}(x, s) ; s \longrightarrow t\} \\
& =\frac{1}{2 \pi i} \int_{c-i \infty}^{c+i \infty} \bar{P}(x, s) e^{-s t} d s, s>0 .
\end{aligned}
$$

Applying the shift theorem and the convolution theorem [16], then the analytical solution with uniformly increasing pollution source $P_{1}(x, t)$ is

$$
\begin{aligned}
P_{1}(x, t)= & \frac{q}{A K_{1}}\left(1-\exp \left(-K_{1} t\right)\right)+\frac{1}{2}\left(P_{0}-\frac{q}{A K_{1}}\right) \exp \\
& \cdot\left(\left(\frac{\beta}{\sqrt{D_{p}}}+\gamma\right) x\right) \operatorname{erfc}\left(\frac{x}{2 \sqrt{D_{p} t}}+\beta \sqrt{t}\right) \\
& +\exp \left(\left(\frac{-\beta}{\sqrt{D_{p}}}+\gamma\right) x\right) \operatorname{erfc}\left(\frac{x}{2 \sqrt{D_{p} t}}-\beta \sqrt{t}\right) \\
& +\frac{q}{2 A K_{1}} \exp \left(\frac{v}{D_{p}} x-K_{1} t\right) \operatorname{erfc} \\
& \cdot\left(\frac{x}{2 \sqrt{D_{p} t}}+\gamma \sqrt{D_{p} t}\right)+\exp \left(-K_{1} t\right) \operatorname{erfc} \\
& \cdot\left(\frac{x}{2 \sqrt{D_{p} t}}-\gamma \sqrt{D_{p} t}\right),
\end{aligned}
$$



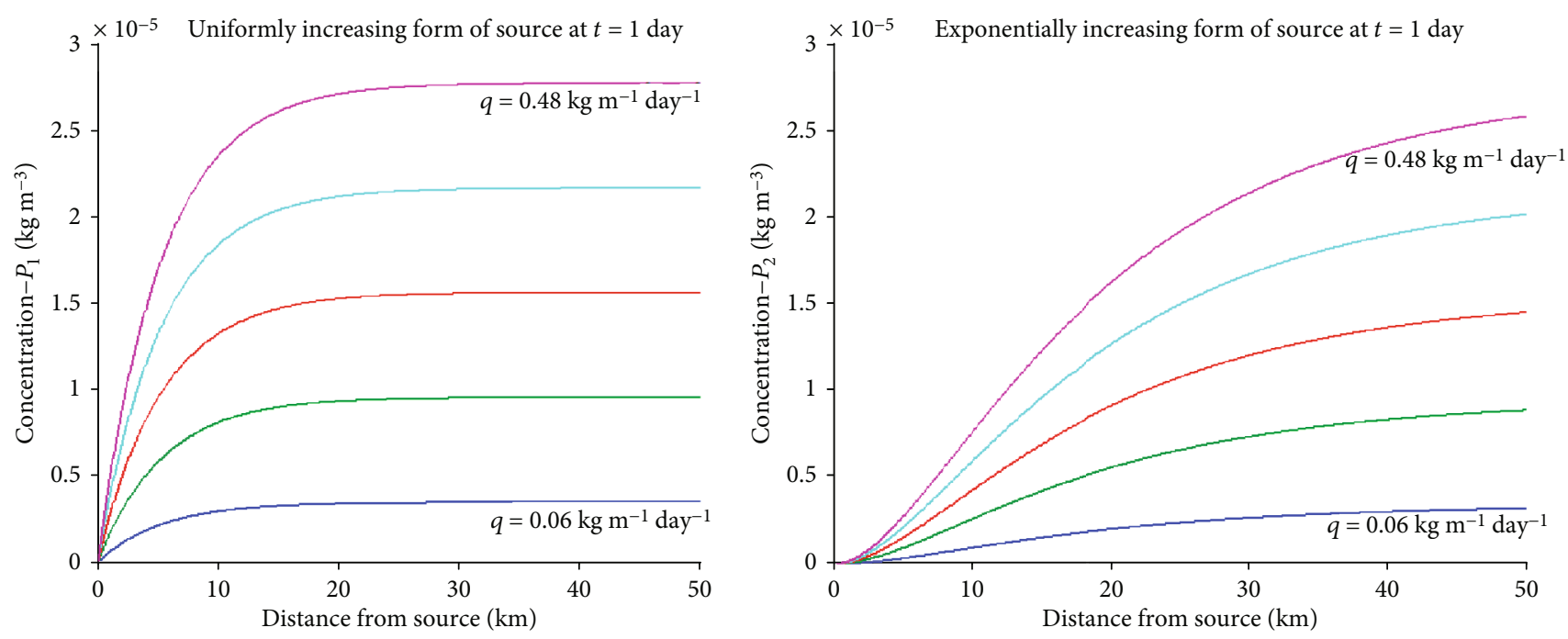

FIGURE 3: Analysis of pollutant concentrations $P_{1}$ and $P_{2}$ of various values of the rate of pollutant addition $(q)$ at $t=1$ day and $0.06 \leq q \leq 0.48$.

and for the analytical solution with exponentially increasing pollution source $P_{2}(x, t)$ is

$$
\begin{aligned}
& P_{2}(x, t)=\frac{q}{A K_{1}}\left(1-\exp \left(-K_{1} t\right)\right) \\
& -\frac{q}{A K_{3}} \exp (-\lambda x)\left(1-\exp \left(-K_{3} t\right)\right) \\
& +\frac{1}{2}\left(P_{o}-\frac{q}{A K_{1}}+\frac{q}{A K_{3}}\right) \exp \\
& \cdot\left(\left(\frac{\beta}{\sqrt{D_{p}}}+\gamma\right) x\right) \operatorname{erfc}\left(\frac{x}{2 \sqrt{D_{p} t}}+\beta \sqrt{t}\right) \\
& +\exp \left(\left(\frac{-\beta}{\sqrt{D_{p}}}+\gamma\right) x\right) \operatorname{erfc}\left(\frac{x}{2 \sqrt{D_{p} t}}-\beta \sqrt{t}\right) \\
& +\frac{q}{2 A K_{1}} \exp \left(\frac{v}{D_{p}} x-K_{1} t\right) \operatorname{erfc} \\
& \left(\frac{x}{2 \sqrt{D_{p} t}}+\gamma \sqrt{D_{p} t}\right)+\exp \left(-K_{1} t\right) \operatorname{erfc} \\
& \cdot\left(\frac{x}{2 \sqrt{D_{p} t}}-\gamma \sqrt{D_{p} t}\right)-\frac{q}{2 A K_{3}} \exp \\
& \left(\left(\frac{\theta}{\sqrt{D_{p}}}+\gamma\right) x-K_{3} t\right) \operatorname{erfc}\left(\frac{x}{2 \sqrt{D_{p} t}}+\theta \sqrt{t}\right) \\
& +\exp \left(\left(\frac{-\theta}{\sqrt{D_{p}}}+\gamma\right) x-K_{3} t\right) \operatorname{erfc} \\
& \cdot\left(\frac{x}{2 \sqrt{D_{p} t}}-\theta \sqrt{t}\right)
\end{aligned}
$$

where $\theta=\sqrt{v^{2} / 4 D_{p}+v \lambda+D_{p} \lambda^{2}}$.
3.2. The Steady State Solution. The steady solution is derived from Equations (20) and (21) by taking limit $t \longrightarrow \infty$. Hence, pollutant concentrations in this state give

$$
\begin{aligned}
P_{1}(x)= & \frac{q}{A K_{1}}+\left(P_{0}-\frac{q}{A K_{1}}\right) \exp \left(\left(\gamma-\frac{\beta}{\sqrt{D_{p}}}\right) x\right), \\
P_{2}(x)= & \frac{q}{A K_{1}}-\frac{q}{A K_{3}} \exp (-\lambda x)+\left(P_{0}-\frac{q}{A K_{1}}\right. \\
& \left.+\frac{q}{A K_{3}}\right) \exp \left(\left(\gamma-\frac{\beta}{\sqrt{D_{p}}}\right) x\right)
\end{aligned}
$$

The downstream pollutant concentration limit were calculated by $x \longrightarrow \infty$ therefore gives

$$
P_{1}(x \longrightarrow \infty, t \longrightarrow \infty)=P_{2}(x \longrightarrow \infty, t \longrightarrow \infty)=\frac{q}{A K_{1}} \text {. }
$$

This limit is the same result which was obtained by Pimpunchat et al. [1].

3.3. Analytical and Numerical Simulation. Numerical solutions are obtained by rearranging Equations (16) and (17); then, the numerical solutions must satisfy

$$
P_{m}^{n+1}=B P_{m+1}^{n}+C P_{m}^{n}+E P_{m-1}^{n}+Q
$$

where $B=D_{P} \Delta t / \Delta x^{2}-v \Delta t / 2 \Delta x, C=1-2 D_{P} \Delta t / \Delta x^{2}-K_{1} \Delta \mathrm{t}$, $E=D_{P} \Delta t / \Delta x^{2}+v \Delta t / 2 \Delta x, Q_{1}=q \Delta t / A$ for uniformly increasing pollution source, and $Q_{2}=q\left(1-\exp \left(-\lambda x_{m}^{n}\right)\right) \Delta t / A$ for 

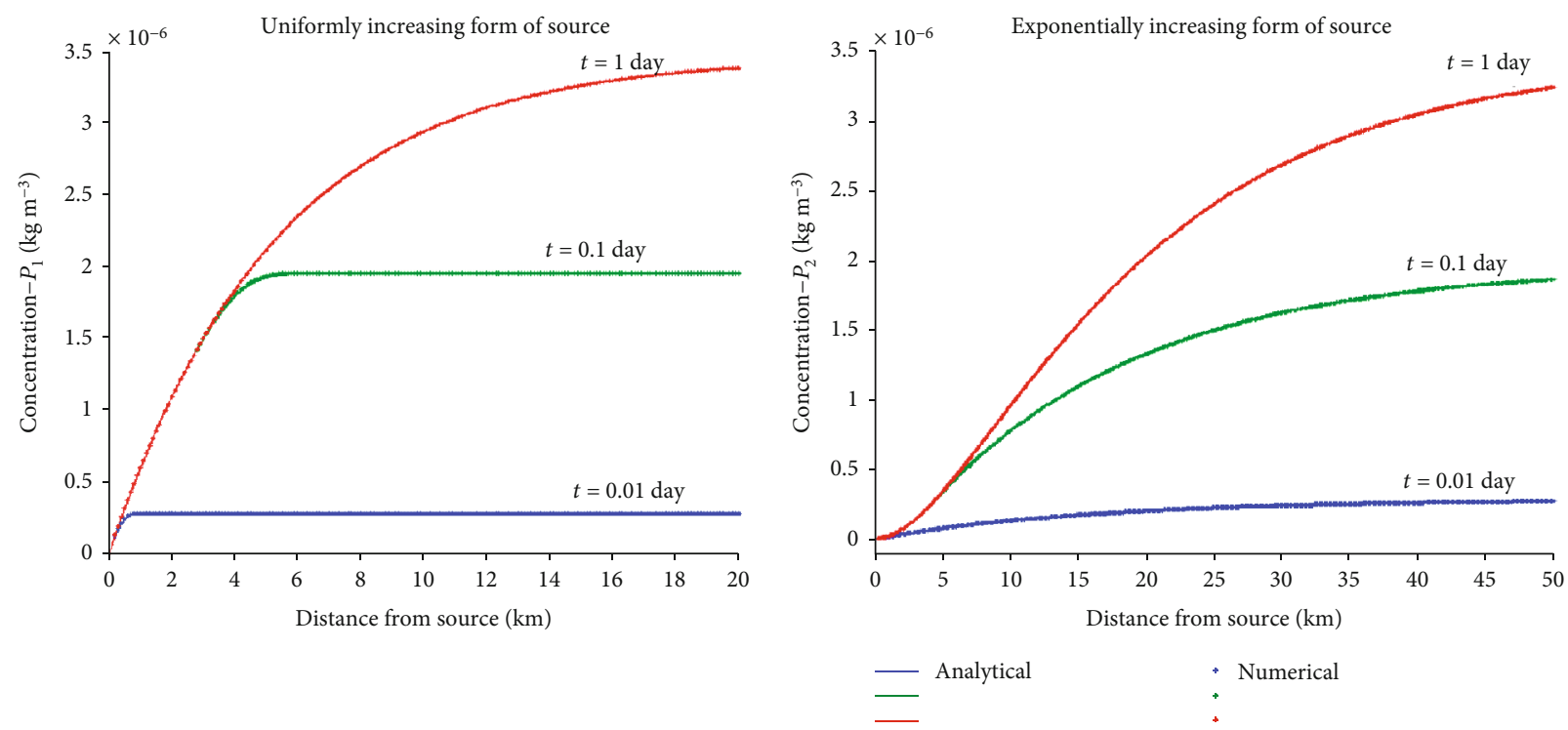

Figure 4: Analytical and numerical solutions of pollutant concentrations $P_{1}$ and $P_{2}$ at $t=0.01,0.1$, and 1 day.

exponentially increasing pollution source. The $x$ and $t$ meshes must be chosen $D_{p} \Delta t /(\Delta x)^{2} \leq 1 / 2$ in order to ensure stability.

$$
\text { Relative error }=\left|\frac{P_{\text {analytical }}-P_{\text {numerical }}}{P_{\text {analytical }}}\right| .
$$

The parameter values used in this study are the same as per Pimpunchat et al. [1]: $D_{p}=3.456 \times 10^{6}\left(\mathrm{~m}^{2}\right.$ day $\left.^{-1}\right), v=$ $4.32 \times 10^{4}\left(\mathrm{~m} \mathrm{day}^{-1}\right), A=2.1 \times 10^{3}\left(\mathrm{~m}^{2}\right), K_{1}=8.27\left(\right.$ day $\left.^{-1}\right)$, and $P_{0}=1 \times 10^{-9}\left(\mathrm{~kg} \mathrm{~m}^{-3}\right)$. Figure 3 shows pollutant concentrations with various rates of pollutant addition along the river by various distance steps $x$ at time $t=1$ day. It starting from $q=0.06$ to $q=0.48\left(\mathrm{~kg} \mathrm{~m}^{-1}\right.$ day $\left.^{-1}\right)$, the subfigure on the left-hand side shows $q$ in the form of uniform increase by Equation (20), and the right-hand side shows $q$ in the form of exponential increase from Equation (21). The arbitrary constant of the exponential pollution source term $(\lambda=$ $0.06289 \mathrm{day}^{-1}$ ) is assumed by the total rate of pollutant addition $q$ being reduced by $5 \%$ along the river $(L=318 \mathrm{~km})$, approximately. At the same position $x$, the result of adding pollutants by different terms makes it clear that the concentration in the right subfigure is less than the concentration in the left subfigure. The concentrations increasingly vary obviously with $q ; P$ increases as $q$ increases. The rate of both concentrations increases rapidly near the origin pollution source and slowly far away. By Equation (6), the concentration gradient at infinity is assumed to be zero; when the distance is long enough, the pollutant concentrations converge to a positive constant. The range of distance at which the concentration is unchanged happens downstream; addition by uniformly increasing pollution source is shorter than addition by exponentially increasing pollution source.

The comparison between the analytical and numerical solutions obtained by the Laplace transform technique and finite different technique is illustrated in Figure 4 by step size
TABLE 1: Relative error of pollutant concentrations by $\Delta x=0.1$ and $\Delta t=0.001$ at $t=1$ day.

\begin{tabular}{lccl}
\hline Distance $(\mathrm{km})$ & $\begin{array}{c}\text { Analytical } \\
\text { solutions }\end{array}$ & $\begin{array}{c}\text { Numerical } \\
\text { solutions }\end{array}$ & Relative errors \\
\hline \multicolumn{4}{c}{ Uniformly increasing pollution source $P_{1}$} \\
5 & $2.10964 \times 10^{-6}$ & $2.10957 \times 10^{-6}$ & $3.48535 \times 10^{-5}$ \\
10 & $2.93091 \times 10^{-6}$ & $2.93085 \times 10^{-6}$ & $1.95423 \times 10^{-5}$ \\
15 & $3.25077 \times 10^{-6}$ & $3.25074 \times 10^{-6}$ & $1.02938 \times 10^{-5}$ \\
20 & $3.37535 \times 10^{-6}$ & $3.37534 \times 10^{-6}$ & $5.14846 \times 10^{-6}$ \\
25 & $3.42387 \times 10^{-6}$ & $3.42387 \times 10^{-6}$ & $2.47106 \times 10^{-6}$ \\
30 & $3.44277 \times 10^{-6}$ & $3.442277 \times 10^{-6}$ & $1.14859 \times 10^{-6}$ \\
\hline & Exponentially increasing pollution source $P_{2}$ \\
5 & $3.52318 \times 10^{-7}$ & $3.52349 \times 10^{-7}$ & $8.67962 \times 10^{-5}$ \\
10 & $9.63291 \times 10^{-7}$ & $9.63313 \times 10^{-7}$ & $2.27143 \times 10^{-5}$ \\
15 & $1.54747 \times 10^{-6}$ & $1.54748 \times 10^{-6}$ & $7.10092 \times 10^{-6}$ \\
20 & $2.02779 \times 10^{-6}$ & $2.02779 \times 10^{-6}$ & $2.07408 \times 10^{-6}$ \\
25 & $2.39946 \times 10^{-6}$ & $2.39946 \times 10^{-6}$ & $3.55824 \times 10^{-7}$ \\
30 & $2.67901 \times 10^{-6}$ & $2.67900 \times 10^{-6}$ & $1.95815 \times 10^{-7}$ \\
\hline
\end{tabular}

$\Delta x=0.1$ and $\Delta t=0.001$. The concentration values are shown in the longitudinal region $0 \leq x \leq 50 \mathrm{~km}$ at different time $t=0.01$ (blue line), $t=0.1$ (green line), and $t=1$ (red line), respectively. The relative errors which are calculated by Equation (25) are provided in Table 1. The relative error is high near the origin and then decreases into a positive value. The maximum percentage relative error is excellent agreement between the analytical and the numerical solutions; it is less than $0.07 \%$ when $x \leq 1 \mathrm{~km}$ and less than $0.05 \%$ when $1<x \leq 5 \mathrm{~km}$, approximately. When the distance is long enough, the concentration values converge into a positive constant, causing the relative errors to gradually decrease 

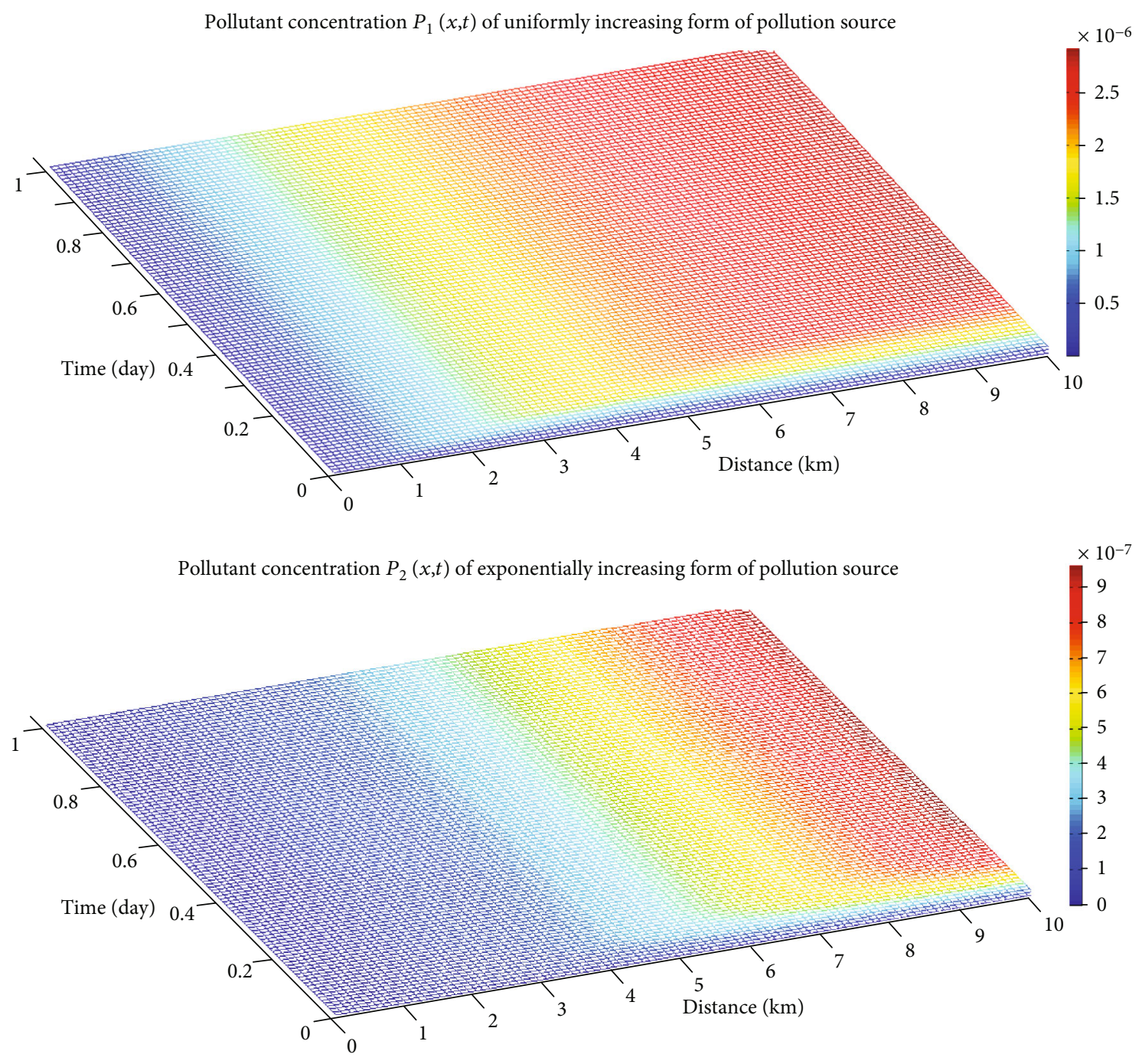

Figure 5: Pollutant concentrations $P_{1}(x, t)$ and $P_{2}(x, t)$ with 100 mesh points by $\Delta x=0.1$ and $\Delta t=0.01$.

into a positive constant value. In addition, pollutant concentration with 100 mesh points is depicted in Figure 5. The graph shows the different levels of increasing concentrations by surface shading.

3.4. Capacity Approximation. The daily load of contamination depends on the term of added pollutant $q$ and the length of river $L$. Certainly, that in case $q$ increases the concentration increases, as depicted by Figure 3. Figure 6 illustrates the behavior of pollutant concentrations $\left(P_{2} / P_{0}\right)$ by vary $\lambda\left(0.25 \times 10^{-5} \leq \lambda \leq 0.46667 \times 10^{-5}\right)$ compared with the observed data between water quality sampling stations TC 15 and TC 13. These stations were chosen because they were separated between the middle and lower parts of rivers and the BOD standard esteem multiplied $(2-4 \mathrm{mg} / \mathrm{L})$. In this manner, $\lambda$ is simulated by the concentration at the end to extend by one time. After that, we utilize these values for estimated assessing the capacity load of pollutant per day between the two stations by $L=36 \mathrm{~km}, q=60$, load is about $2,160 \mathrm{~kg}$ per day by uniformly incremental but the daily load of the exponentially is less than up to $90 \%$ which appeared approximation computation these values by Table 2. By this simulation, we can offer at the same rate $q$ the analytical solution with exponentially increasing pollution source as a suitable model for illustrating the behavior of incremental pollution along the river.

3.5. Variable Coefficients. In our model, the medium of transport has a uniform dispersion and velocity. To apply our model to the case of spatially temporally dependent coefficients, it can therefore be modeled with variable coefficients as

$\frac{\partial(A P)}{\partial t}=\frac{\partial}{\partial x}\left[D_{p}(x, t) \frac{\partial(A P)}{\partial x}-v(x, t) A P\right]-K_{1} A P+q H(x)$,

let $D_{p}(x, t)=D_{p} f_{1}(x, t), v(x, t)=v_{0} f_{2}(x, t), f_{1}(x, t)=f$ $(m t)$, and $f_{2}(x, t)=1$ [10]. This model applies to temporally dependent dispersion along a uniform flow. By these transformations, $d X / d x=-1 / f_{1}(x, t)$ or $X=\int d x / f_{1}(x, t)$ and $T=$ 


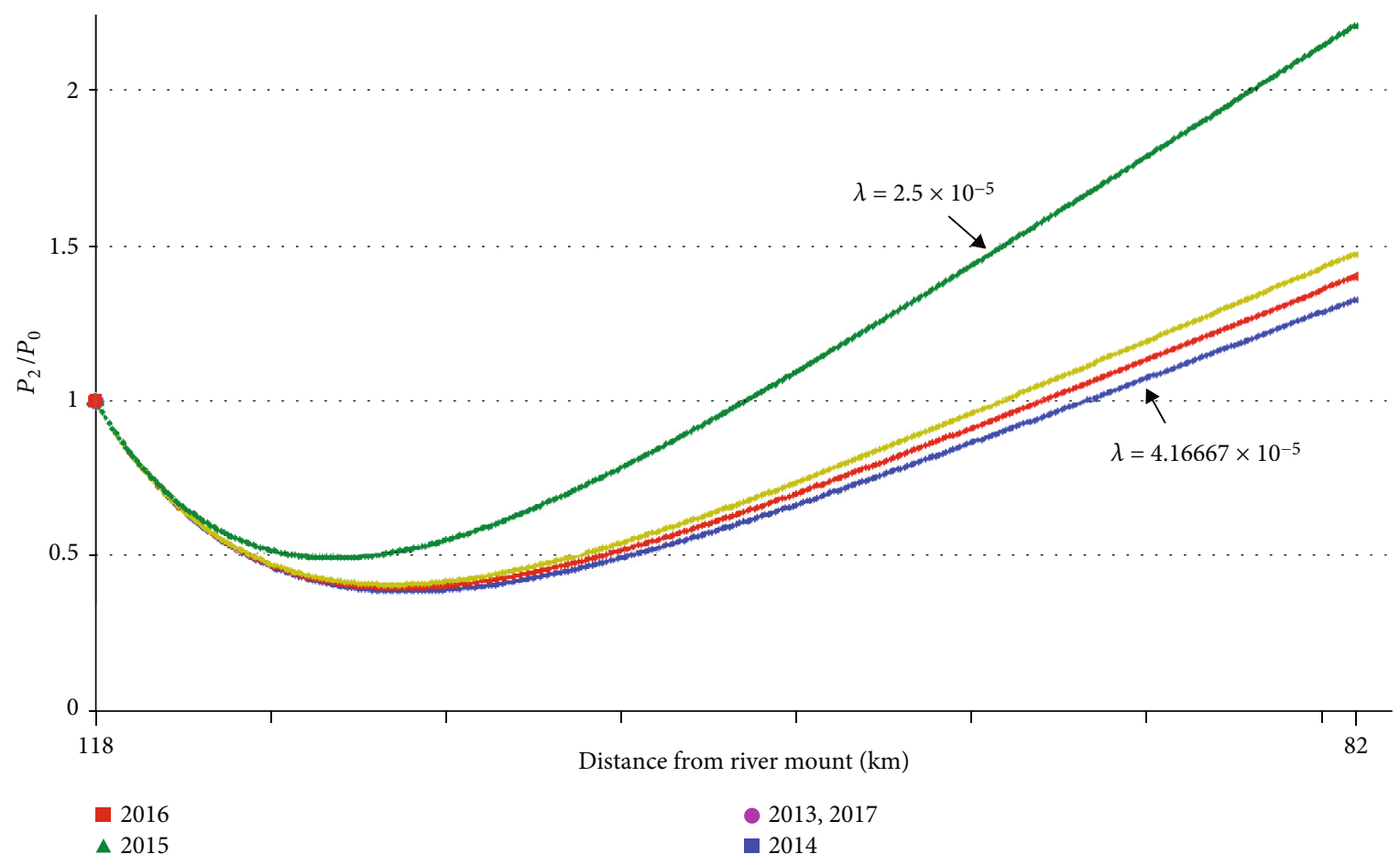

FIGURE 6: Behaviors of pollutant concentration $\left(P_{2} / P_{0}\right)$ at $t=1$ day by various arbitrary constants of exponentially increasing pollution source terms $\left(0.25 \times 10^{-5} \leq \lambda \leq 0.46667 \times 10^{-5} \mathrm{day}^{-1}\right)$ with $q=60 \mathrm{~kg} / \mathrm{km}$ day.

$\int_{0}^{t} d t / f(m t)$ (Crank (1975)); then, Equation (26) reduces to our model Equation (3) $\partial(A P) / \partial T=D_{p}\left(\partial^{2}(A P) / \partial X^{2}\right)+v_{0}$ $(\partial(A P) / \partial X)-K_{1} A P+q$, where $D_{p}$ and $v_{0}$ are constants in which the analytical solution can be obtained by using the Laplace transform technique the same with this work.

In addition, the model can apply to spatially dependent dispersion along a nonuniform flow by $D_{p}(x, t)$ is considered proportional to the square of $v(x, t)$ instead of proportion to $v(x, t)$. Both terms in Equation (26) are obtained as follows: $f_{1}(x, t)=(1+a x)^{2}$ and $f_{2}(x, t)=(1+a x)$, where $a x$ is nondimensional and $a$ is nonzero real constant accounting for the variations in velocity and dispersion due to inhomogeneity [10]. From previous transformation, we will have another spatial variable $X=1 / a(1+a x)$ then Equation (26) reduces to

$$
\begin{aligned}
\frac{\partial(A P)}{\partial t}= & a^{2} D_{p} X^{2} \frac{\partial^{2}(A P)}{\partial X^{2}}+a v_{0} X \frac{\partial(A P)}{\partial X} \\
& -\left(a v_{0} X+K_{1}\right) A P+q .
\end{aligned}
$$

To evaluate the analytical solution of Equation (27), additional studies can be conducted in this work and the previous work [10]. Another case for the cross-section area in Equation (3) vary with position $x$, and we may get the desired equation as

$$
\frac{\partial P}{\partial t}=D_{p} \frac{\partial^{2} P}{\partial x^{2}}-v \frac{\partial P}{\partial x}-K_{1} P+\frac{q}{A(x)}
$$

\begin{tabular}{|c|c|c|}
\hline \multicolumn{3}{|c|}{ Exponentially increasing pollution source $L=36 \mathrm{~km}$ and $q=60$} \\
\hline$\lambda$ & $\begin{array}{l}P_{2} / P_{0} \text { at the end } \\
\text { numerical solutions }\end{array}$ & $\begin{array}{c}\text { Daily load (kg/day) } \\
\text { difference (\%) }\end{array}$ \\
\hline $2.5 \times 10^{-5}$ & 1.32439 & $99.95501 \%$ \\
\hline $2.63889 \times 10^{-5}$ & 1.39788 & $99.52516 \%$ \\
\hline $2.77778 \times 10^{-5}$ & 1.47136 & $99.95002 \%$ \\
\hline $4.16667 \times 10^{-5}$ & 2.20598 & $99.92504 \%$ \\
\hline
\end{tabular}

TABLE 2: Various $\lambda$ of exponentially increasing pollution source for $P_{2 /} P_{0}$ concentration and daily load difference.

in which the analytical solutions can be obtained by using Laplace transformation techniques in this work as well.

\section{Concluding Remarks}

The unsteady state solutions of pollutant concentration by considering advection-dispersion equations in 1-D are proposed by using the Laplace transform technique and the explicit finite difference technique, for analytical and numerical solutions, respectively. The model is presented by considering the rate of pollutant addition along the river by uniformly increasing and exponentially increasing forms. The trend of concentrations is expanded with time and space, which is affected by the rate of pollutant addition along the river $q$. It is obviously found that the limits of the downstream concentrations that are increased $q$ by the exponentially increasing form are less than the increased $q$ by the uniformly increasing form, which is the result of the behavior of added pollutant sources. In reality, this model is suitable for the river which has pollution source that varies with 
position by the pollution sources in the downstream which is higher than the downstream, as appeared within the Tha Chin river and the Chao Praya River in Thailand. The source of contamination of the case study river will be spread from the center to the end, causing the concentration of contamination will continuously increment. The arbitrary constant $\lambda$ was presented and illustrated to significantly compare the behavior of exponential increasing form of sources. The solutions of this study can be used to predict the effect of added pollutants on the maximum allowable loads of pollutants that a river can have from all sources by changing the value of $q$ and $\lambda$. The results can be applied in many physical situations described by advection-dispersion phenomena and in making decisions to support the planning and managing of river water quality issues.

\section{Data Availability}

The result of this study was from Analytical and Numerical techniques. We did not use the raw data.

\section{Conflicts of Interest}

The authors declare that they have no conflicts of interest.

\section{Acknowledgments}

The first author is grateful to King Mongkut's University of Technology North Bangkok for the financial support during her study doctor of philosophy. Finally, the authors would like to express their gratitude to the anonymous referees for very helpful suggestions and comments which led to improvements of the original manuscript.

\section{References}

[1] B. Pimpunchat, W. L. Sweatman, G. C. Wake, W. Triampo, and A. Parshotam, "Modelling river pollution and removal by Aeration," in MODSIM 2007 International Congress on Modelling and Simulation. Land, Water \& Environment Management: Integrated Systems for Sustainability, L. Oxley and D. Kulasiri, Eds., pp. 2431-2437, Modelling and Simulation of Australia and New Zealand, 2007.

[2] B. Pimpunchat, W. L. Sweatman, G. C. Wake, W. Triampo, and A. Parshotam, "A mathematical model for pollution in a river and its remediation by aeration," Applied Mathematic Letters, vol. 22, no. 3, pp. 304-308, 2009.

[3] W. Simachaya, Water Quality Management in ThailandPollution Control Department (PCD), http://www.pcd.go.th/info_ serv/water.html.

[4] W. Simachaya, "A decade of water quality monitoring in Thailand's four major rivers: the results and the implications for management," in Proceedings of the 6th International Conference on the Environmental Management of Enclosed Coastal Seas, Bangkok, Thailand, 2003a.

[5] N. Singkran, "Determining water conditions and carrying capacity of the Tha Chin river," in The 3rd Technology and Innovation for Sustainable Development International Conference, Royal Mekong Nongkhai Hotel, Nong Khai, Thailand, Faculty of Engineering, Khon Kaen University, 2010, Thailand (Best Paper Award).
[6] Regional Environmental office 5, Mobile application Tha Chin water qualityMinistry of Natural Resources and Environmenthttp://www.mnre.go.th/reo05/th/news/detail/22702.

[7] C. Wongsupap, S. Weesakul, R. Clemente, and A. Das Gupta, "River basin water quality assessment and management: case study of Tha Chin river basin, Thailand," Water International, vol. 34, no. 3, pp. 345-361, 2009.

[8] Pollution Control Department (PCD), Water quality standards \& criteria in Thailand (in Thai), Ministry of Science, Technology and Environment, Thailand, 4 edition, 2000.

[9] M. T. Van Genuchten and W. J. Alves, "Analytical solutions of the one-dimensional convective-dispersive solute transport equation," in Technical Bulletin, vol. 1661, US Department of Agriculture, 1982.

[10] A. Kumar, D. K. Jaiswal, and N. Kumar, "Analytical solutions to one-dimensional advection-diffusion equation with variable coefficients in semi-infinite media," Journal of Hydrology, vol. 380, no. 3-4, pp. 330-337, 2010.

[11] S. Savović and A. Djordjevich, "Finite difference solution of one- dimensional advection-dispersion equation with variable coefficients in semi-infinite media," International Journal of Heat and Mass Transfer, vol. 55, pp. 4291-4294, 2012.

[12] W. E. Dobbins, "BOD and oxygen relationships in streams," Journal of the Sanitary Engineering Division, vol. 90, no. 3, pp. 53-78, 1964.

[13] S. C. Chapra, Surface Water Quality Modeling, The McGrawHill Company, 1997.

[14] N. Ian Sneddon, The Use of Integral Transforms, TAtA McGraw-Hill Publishing Company LTD, New Delhi, 1974.

[15] D. Murat, "On an application of Laplace transforms," New Trends in Mathematical Science, vol. 5, no. 2, pp. 193-198, 2017.

[16] G. E. Roberts and H. Kaufman, Table of Laplace Transforms, W.E. Saunders Co., Philadelphia and London, 1969. 